\title{
Review of: "Ferroelectric 2D ice under graphene confinement"
}

\author{
Giancarlo Franzese ${ }^{1}$ \\ 1 University of Barcelona
}

Potential competing interests: The author(s) declared that no potential competing interests exist.

\section{Supercomputer memories made of water.}

How can water help us get computers with a memory large enough to reboot them in a second? A work published in Nature Communications in November 2021--by researchers from the Taiwan Academia Sinica, the National Taiwan University, the Taiwan National Cheng Kung University, the Taiwan National Chung Cheng University, and the Czech Republic Charles University--shows the way. The authors study what happens to the water due to ambient humidity between two graphene sheets that almost touch each other.

The team observes that, at ambient temperature, the water in the graphene pore has an intense and permanent electromagnetic dipole that depends on a previously applied voltage. This property of water is called ferroelectricity and has been discussed before in literature. Previous works showed that, at ambient temperature, a water monolayer, confined between two graphene layers, forms hexagonal ice. Here the authors show that, under the action of the applied voltage, the monolayer ice orients its molecules along a preferential direction, transforming into a new ice phase (ice $\mathrm{XI}$ ). They discuss how to control this transformation by applying a voltage difference that changes the separation between the graphene sheets.

They create multiple drum structures with two graphene layers. By increasing the voltage difference between them, the two sides of the drum get in contact by electrostatic attraction. The reduction of the available volume squeezes out the water up to leaving only a monolayer around the contact point of each drum. This nanoelectromechanical actuation approach provides fine-tuning of the local formation of a water monolayer, something hard to do in general.

The molecular orientation does not occur spontaneously but at a defined positive voltage $(\sim 5 \mathrm{~V})$, with a switching behavior marked by low resistance to electron tunneling between the two graphene layers. Surprisingly, the ice persists in its polarized state even if the voltage is turned off (remanent polarization). Hence, the device works as non-volatile memory. 
Also, the team finds that the polarization goes to zero only under the action of a negative voltage, showing a hysteresis curve typical of a memory device. The larger the polarizing positive voltage, the larger the unpolarizing negative voltage. This property allows the authors to set four different states in each drum, realizing a stable and fast 4-bit multi-level mechanical data storage device. Its dynamic relationship between current and voltage, including a memory of their past values, makes it a ferroelectric memristor. Potential applications go from analog memories for quantum computers to solid-state memories storing the data of 100 trillion modern flash memories in just one $\mathrm{cm}^{3}$ at the same reading speed.

An intriguing aspect of this study is that the switching behavior disappears for confined multilayers of water. Why? The answer comes from a theoretical work published in ACS Nano at the same time as the experimental one (November 2021) within a collaboration between the Rome University La Sapienza and the University of Barcelona. This work shows that a water monolayer confined between graphene sheets is slightly less stable than a bilayer but is drastically different. Indeed, the bilayer stability is due to the many hydrogen bonds, as in multilayers, between the water molecules. Once formed, their breaking costs energy. Instead, in the monolayer, the water molecules point toward the center of the pore, inhibiting the hydrogen bonds and forming a disordered structure. Hence, an applied field can re-orient the molecules in the monolayer at much less cost than in the bilayer. This observation clarifies why we can observe the memristor switching behavior in monolayer water but not in multilayer water ice.

Ferroelectric 2D ice under graphene confinement. Hao-Ting Chin, Jiri Klimes, I-Fan Hu, Ding-Rui Chen, Hai-Thai Nguyen, Ting-Wei Chen, Shao-Wei Ma, Mario Hofmann, Chi-Te Liang, Ya-Ping Hsieh, Nat. Commun. 2021, 12, 6291

https://doi.org/10.1038/s41467-021-26589-x

Nanoconfined Fluids: Uniqueness of Water Compared to Other Liquids. Fabio Leoni, Carles Calero, and Giancarlo Franzese,

ACS Nano 2021, 15, 12, 19864-19876

https://doi.org/10.1021/acsnano.1c07381 